\title{
Neutrino Opacity in High Density Nuclear Matter
}

\author{
Sérgio M. dos Santos*, Moisés Razeira*, Lev Leinson ${ }^{\dagger}$, César A.Z. \\ Vasconcellos*, Bardo E.J. Bodmann** and Manfred Dillig ${ }^{*}$ \\ *Instituto de Física, Universidade Federal do Rio Grande do Sul, CEP 91501-970 Porto Alegre, \\ Rio Grande do Sul, Brazil \\ ${ }^{\dagger}$ Institute of Terrestrial Magnetism, Ionosphere and Radio Wave Propagation, 142190 Troitsk, \\ Moscow Region, Russia \\ **Instituto de Física e Matemática, Universidade Federal de Pelotas, CEP 96010-900 Pelotas, Rio \\ Grande do Sul, Brazil \\ ${ }^{\ddagger}$ Institut für Theoretische Physik III, der Universität Erlangen-Nürnberg, D91058 Erlangen, \\ Germany
}

\begin{abstract}
We estimate the contribution of the nucleon weak magnetism on the neutrino absorption mean free path inside high density nuclear matter. In the mean field approach, three different ingredients are taken into account: (a) a relativistic generalization of the approach developed by Sanjay et al.; (b) the inclusion of the nucleon weak-magnetism (c) and the pseudo-scalar interaction involving the nucleons. Our main result shows that the neutrino absorption mean free path is three times the corresponding result obtained by those authors.
\end{abstract}

\section{NEUTRINO OPACITY IN NEUTRON STARS}

Neutrino opacity is an important issue in the study of the evolution of protoneutron stars. The cross section per unit volume of matter for neutrinos in high density matter is derived from the Fermi's golden rule[1]; the transition rate for neutrino scattering and absorption by nucleons[1] is determined by the sum over the final spins and average over initial spins and by taking into account the nucleon distribution functions $\left(f_{i}\right)$ for thermal equilibrium. For baryon densities $n_{B} \leq 5 n_{0}$, where $n_{0}=0.16 \mathrm{fm}^{-3}$ is the empirical nuclear equilibrium density, and in the absence of interactions baryons may be considered as non-relativistic. Under this condition, the transition rate becomes a constant, $W_{f i}=G_{F}^{2}\left(\mathscr{V}^{2}+3 \mathscr{A}^{2}\right)$ with $\mathscr{V}$ and $\mathscr{A}$ denoting the vector and axial coupling constants. The free gas isospin density response function is given as[1] $S\left(q_{0}, q\right)=$ $\frac{M^{2} T}{\pi q} \frac{z}{1+\exp (-z)}\left(1+\frac{\xi_{-}}{z}\right)$, where $\xi_{-}=\ln \frac{1+\exp \left(\frac{e_{-}-\mu_{2}}{T}\right)}{1+\exp \left(\frac{e_{-}+q_{0}-\mu_{4}}{T}\right)} ; e_{-}=\frac{p_{-}^{2}}{2 M}=\frac{1}{4} \frac{\left(q_{0}-q^{2} / 2 M\right)^{2}}{q^{2} / 2 M}$. An expression for the cross section per unit volume of matter for both scattering and absorption is then $\frac{\sigma\left(E_{1}\right)}{V}=G_{F}^{2}\left(\mathscr{V}^{2}+3 \mathscr{A}^{2}\right) \int \frac{d^{3} p_{3}}{(2 \pi)^{3}}\left(1-f_{3}\left(E_{3}\right)\right) S\left(q_{0}, q\right)$. This result, which we further generalize to include nuclear interactions, proves to be the key ingredient for efficient calculations of neutrino opacities. The cross section per unit volume of matter 
for neutrino scattering and absorption by nucleons is given by[1]

$$
\begin{aligned}
\frac{\sigma\left(E_{1}\right)}{V} & =2 \int \frac{d^{3} p_{2}}{(2 \pi)^{3}} \int \frac{d^{3} p_{3}}{(2 \pi)^{3}} \int \frac{d^{3} p_{4}}{(2 \pi)^{3}}(2 \pi)^{4} \delta^{4}\left(P_{1}+P_{2}-P_{3}-P_{4}\right) \frac{\left\langle|\mathscr{M}|^{2}\right\rangle}{2^{4} E_{1} E_{2} E_{3} E_{4}} \\
& \times f_{2}\left(E_{2}\right)\left(1-f_{3}\left(E_{3}\right)\right)\left(1-f_{4}\left(E_{4}\right)\right),
\end{aligned}
$$

where $P_{i}=\left(E_{i}, \vec{p}_{i}\right)$ denotes the four-momentum of particle $i$. We extend the noninteracting and non-relativistic approach[1] considering, the relativistic absorption process $n\left(v_{e}, e\right) p$ in nuclear matter.

$$
\begin{aligned}
\mathscr{M}_{f i}= & -i \frac{G_{F} C}{\sqrt{2}} \bar{u}\left(k_{2}\right) \gamma_{\mu}\left(1+\gamma_{5}\right) v\left(-k_{1}\right) \bar{u}_{p}\left(P^{\prime}\right)\left[C_{V} \gamma^{\mu}\right. \\
& \left.+\frac{C_{M}}{2 M} \sigma^{\mu v} q_{v}+C_{A}\left(\gamma^{\mu} \gamma_{5}+F_{q} q^{\mu} \gamma_{5}\right)\right] u-n(P),
\end{aligned}
$$

where $C_{V}=1 ; C_{M}=\lambda_{p}-\lambda_{n} 3.7 ; C_{A}=1.26$.

The nuclear matter equation of state at finite temperature is determined by a parameter set that reproduces the nucleon effective mass $M^{*}=700 \mathrm{MeV}$, at saturation density $\left(\rho_{0}=\right.$ $0.6 \mathrm{fm}^{-3}$ ), the binding energy $B=-16 . \mathrm{MeV}$, the compression modulus $\mathrm{K}=300 \mathrm{MeV}$ and the symmetry energy $a_{4}=32.5 \mathrm{MeV}$. The lagrangian density of our approach is defined for the baryon octet $\left(B=p, n, \Lambda, \Sigma^{-}, \Sigma^{0}, \Sigma^{+}, \Xi^{-}, \Xi^{0}\right)$ coupled to three mesons $(\sigma, \omega, \rho)$ and leptons.

$$
\begin{aligned}
\mathscr{L} & =\sum_{B} \bar{\psi}_{B}\left[\left(i \gamma_{\mu}\left(\partial^{\mu}-g_{\omega B} \omega^{\mu}\right)-\left(M_{B}-g_{\sigma B} \sigma\right)\right] \psi_{B}-\sum_{B} \bar{\psi}_{B}\left[\frac{g_{\rho B}}{2} \tau \cdot \rho^{\mu}\right] \psi_{B}\right. \\
& +\frac{b M}{3} \sigma^{3}+\frac{c}{4} \sigma^{4}+\frac{1}{2}\left(\partial_{\mu} \sigma \partial^{\mu} \sigma-m_{\sigma}^{2} \sigma^{2}\right)-\frac{1}{4} \omega_{\mu \nu} \omega^{\mu \nu}+\frac{m_{\omega}^{2}}{2} \omega_{\mu} \omega^{\mu} \\
& -\frac{1}{4} \rho_{\mu \nu} \cdot \rho^{\mu v}+\frac{m_{\rho}^{2}}{2} \rho_{\mu} \cdot \rho^{\mu}+\sum_{l} \bar{\psi}_{l}\left[i \gamma_{\mu} \partial^{\mu}-M_{l}\right] \psi_{l} .
\end{aligned}
$$

The hyperon/nucleon coupling constant ratios are determined through the binding energy of the $\Lambda$-hyperon which correlates the values of the hyperon/ $\sigma, \omega)$ coupling constants $\chi_{\sigma}$ and $\chi_{\omega}:(B / A)=\chi_{\omega} V-\chi_{\sigma} S$, where $V=\left(g_{\omega} / m_{\omega}\right)^{2} \rho_{0}$ is the mean-field vector potential and $S=\left(m-m_{s a t}^{\star}\right)$ is the mean-field scalar potential. Trapped neutrinos at chemical equilibrium are included following the lepton fraction determination in the study of the gravitational collapse of the core of white dwarfs. The lepton fraction is defined as the ratio $Y_{L}=\left(\rho_{e}+\rho_{v}\right) / \rho_{B}$, where $\rho_{e}, \rho_{v}$ and $\rho_{B}$ are the electron, neutrino and baryon densities, respectively. The equation of state for nuclear matter is obtained as a parametric equation $p=p(\varepsilon)$ which relates the energy density and pressure considering the Fermi-Dirac distribution functions for particle and anti-particles at finite temperature. The chemical potential for particle $i$ is given by $\mu_{i}^{*}=\mu_{i}-\chi \omega i \frac{g_{\omega}^{2}}{m_{\omega}^{2}} \rho_{B}-\chi_{\rho i} I_{3, i} \frac{g_{\rho}^{2}}{m_{\rho}^{2}} \rho_{3}$.

We then apply the Sommerfeld approximation[2] and obtain the finite temperature expressions for the energy density, pressure and chemical potential as deviations of the zero temperature results, $\varepsilon_{0}, p_{0}$ and $\mu_{0}$

$\varepsilon=\varepsilon_{0}+\sum_{i=B, \ell} \frac{\gamma\left(k_{B} T\right)^{2}}{12}\left[2 k_{F, i} E_{F, i}+\frac{k_{F, i}^{3}}{E_{F, i}}\right]+\frac{\gamma \pi^{2}\left(k_{B} T\right)^{4}}{720}\left[\frac{12 k_{F, i}}{E_{F, i}}-9\left(\frac{k_{F, i}}{E_{F, i}}\right)^{3}+3\left(\frac{k_{F, i}}{E_{F, i}}\right)^{5}\right] \ldots$, 


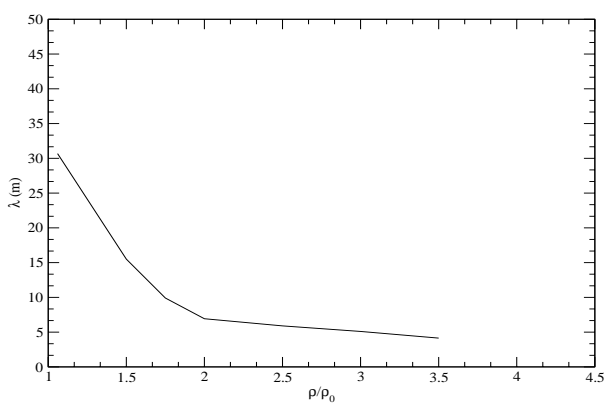

FIGURE 1. Neutrino absorption mean free path in dense nuclear matter at $T=5 \mathrm{MeV}$.

$$
\begin{gathered}
p=p_{0}+\sum_{i=B, \ell} \frac{\gamma\left(k_{B} T\right)^{2}}{12}\left[\frac{4 k_{F, i}^{3}}{E_{F, i}}-\frac{k_{F, i}^{5}}{E_{F, i}^{3}}\right] \\
+\frac{\gamma \pi^{2}\left(k_{B} T\right)^{4}}{720}\left[\frac{24 k_{F, i}}{E_{F, i}}-39\left(\frac{k_{F, i}}{E_{F, i}}\right)^{3}+9\left(\frac{k_{F, i}}{E_{F, i}}\right)^{5}+15\left(\frac{k_{F, i}}{E_{F, i}}\right)^{7}\right]+\ldots, \\
\text { and } \mu=\mu_{0}\left[1-\frac{1}{3}\left(\frac{\pi \kappa T}{2 E_{F}}\right)^{2}\right]
\end{gathered}
$$

\section{RESULTS AND CONCLUSIONS}

In the comparison with the work developed by Sanjay and collaborators, our results indicate the relevance of the nucleon weak magnetism in the neutrino absorption mean free path in high density nuclear matter. In spite of more involved calculations being necessary to improve our knowledge about the relevance of neutrino opacities in the study of the evolution and cooling of protoneutron stars as for instance taking into account different equations of state for dense nuclear matter, our predictions[3] for the neutrino absorption mean free path is three times the corresponding result obtained by Sanjay and collaborators[1].

\section{REFERENCES}

1. Sanjay Reddy, Madappa Prakash, James M. Lattimer, Phys. Rev. D58, 013009 (1998).

2. G.F. Marranghello, C.A.Z. Vasconcellos, M. Dillig and J.A. de Freitas Pacheco, Int. J. Mod. Phys. E11 (2002) 83.

3. C.A.Z. Vasconcellos et al. (2004) (to be published). 
Copyright of AIP Conference Proceedings is the property of American Institute of Physics and its content may not be copied or emailed to multiple sites or posted to a listserv without the copyright holder's express written permission. However, users may print, download, or email articles for individual use. 